\title{
Découverte de la mandibule d'un jeune enfant dans un niveau gravettien de la grotte de Gargas (Hautes-Pyrénées, France)
}

Pascal Foucher, Cristina San Juan-Foucher, Dominique Henry-Gambier, Carole Vercoutère et Catherine Ferrier

\section{(2) OpenEdition \\ Journals}

Édition électronique

URL : http://journals.openedition.org/paleo/2428

DOI : $10.4000 /$ paleo.2428

ISSN : 2101-0420

Éditeur

SAMRA

Édition imprimée

Date de publication : 15 décembre 2012

Pagination : 323-336

ISSN : $1145-3370$

\section{Référence électronique}

Pascal Foucher, Cristina San Juan-Foucher, Dominique Henry-Gambier, Carole Vercoutère et Catherine Ferrier, "Découverte de la mandibule d'un jeune enfant dans un niveau gravettien de la grotte de Gargas (Hautes-Pyrénées, France) », PALEO [En ligne], 23 | 2012, mis en ligne le 29 avril 2013, consulté le 27 juillet 2020. URL : http://journals.openedition.org/paleo/2428 ; DOI : https://doi.org/ $10.4000 /$ paleo.2428

Ce document a été généré automatiquement le 27 juillet 2020

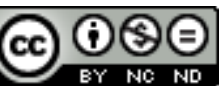

PALEO est mis à disposition selon les termes de la licence Creative Commons Attribution - Pas d'Utilisation Commerciale - Pas de Modification 4.0 International. 


\title{
Découverte de la mandibule d'un jeune enfant dans un niveau gravettien de la grotte de Gargas (Hautes-Pyrénées, France)
}

\author{
Pascal Foucher, Cristina San Juan-Foucher, Dominique Henry-Gambier, \\ Carole Vercoutère et Catherine Ferrier
}

\section{1 - Introduction}

1 La grotte de Gargas, site pyrénéen connu par son riche ensemble pariétal (fig. 1), a fait l'objet de nouvelles fouilles depuis 2004. Celles-ci interviennent un siècle après les dernières recherches archéologiques conduites sur le site par É. Cartailhac et H. Breuil en 1911 et $1913^{1}$.

2 Le principal objectif de cette opération est l'obtention d'une séquence stratigraphique détaillée du gisement (Foucher et al. 2008c) et la réactualisation des données anciennes des fouilles Cartailhac-Breuil (Breuil, Cheynier 1958). A terme, ces informations contribueront à l'élaboration d'une synthèse régionale sur le Gravettien du sud-ouest de l'Europe (Foucher 2004, 2006 et à paraître ; Foucher et al. 2008c; Foucher et al. 2011 ; San Juan-Foucher 2011 et à paraître).

3 Cette reprise des recherches de terrain répond également à une problématique globale incluant la révision des collections Cartailhac-Breuil et une approche de la cavité qui prenne en compte de façon intégrée le double aspect grotte ornée et site d'habitat. On souhaite ainsi mieux comprendre les caractéristiques fonctionnelles, spatiales et chronologiques de la fréquentation du site par les auteurs des manifestations pariétales (Foucher et al. 2007, 2012).

4 Après avoir procédé à l'élaboration d'une synthèse préalable des données historiques des fouilles (Foucher et al. 2007), destinée à mieux orienter nos choix, nous avons ouvert trois sondages dans la grotte inférieure de Gargas (fig. 2). Le premier (GES) se 
situe en limite des anciennes fouilles Cartailhac-Breuil, dans la partie distale du cône d'éboulis qui a obturé l'entrée préhistorique ; le second (GPO) est localisé dans la zone proximale de cet éboulis, à une quinzaine de mètres en amont du premier sondage, dans une salle correspondant au vestibule de l'ancien porche préhistorique; le troisième (GPA) se situe au centre de la Salle I, plus proche de la Grande Paroi des Mains et à proximité du deuxième sondage Cartailhac-Breuil (Foucher et al. 2008c; Foucher, San Juan-Foucher 2011, dir.). C'est dans ce dernier sondage que la mandibule d'enfant a été découverte.

\section{2 - Localisation et cadre sédimentologique de la découverte}

\section{a - Position topographique du sondage GPA}

5 Afin de mieux définir les relations stratigraphiques entre la zone d'habitat fouillée anciennement et la paroi ornée du « Panneau principal des Mains », nous avons ouvert, en 2009, un nouveau secteur de fouille (GPA) dans la zone centrale de la Salle I (fig. 2 et 3). À cet endroit, le sol de la Galerie des Colonnes qui donne accès à la partie profonde de la cavité, en très légère pente (environ $2^{\circ}$ ), rejoint celui de la Salle I, subhorizontal. Les ruissellements d'eau, alimentés par les infiltrations au travers des fissures du plafond, et l'inclinaison du sol de la galerie sont à l'origine du développement d'une succession de gours étagés, actifs après de fortes pluies.

6 Le sondage GPA se trouve également au pied d'une grande stalagmite, prolongée par un plancher stalagmitique incliné de $30^{\circ}$ environ (fig. 4). La moitié de la base de cette stalagmite avait été éventrée par les anciens fouilleurs pour accéder au niveau gravettien, partiellement fouillé en sape. Cette zone se trouve à proximité d'un des sondages de E. Cartailhac et $\mathrm{H}$. Breuil, mais les traces d'excavation encore visibles sont probablement celles de fouilleurs postérieurs, restés anonymes. À l'heure actuelle, on peut observer, sous cette stalagmite, des placages résiduels de sédiments noirs, riches en matériels archéologiques de toute nature (os brûlés, vestiges de faune variée, éclats de silex, etc.) ainsi qu'une formation à galets attribuables à de vieilles alluvions karstiques.

\section{b - Description sédimentologique du sondage GPA}

\section{Couche 1 : plancher stalagmitique (l'épaisseur varie entre $2-3 \mathrm{~cm}$ et $20 \mathrm{~cm}$ )}

7 Le concrétionnement développé à la surface du niveau archéologique résulte des ruissellements provenant de la stalagmite située à proximité et de la Galerie des Colonnes (fig. 4). Ces deux apports peuvent expliquer les différences observées dans la structure du plancher: dans le secteur alimenté par la concrétion, la calcite est compacte et bien cristallisée ; en revanche, dans la zone liée aux gours, elle est friable et comprend un mince niveau argileux résultant vraisemblablement de la décantation de particules transportées en suspension. 
Couche 2 : niveau archéologique - Gravettien (niveau en cours de fouille, de $20 \mathrm{~cm}$ d'épaisseur)

8 C'est un dépôt à support clastique fermé ou semi-ouvert. Il est constitué par des blocs, des cailloux et des granules de calcaire en plaquette ou de forme polyédrique et par des galets de roches endogènes. Les esquilles d'os, brûlées ou non, sont très nombreuses. Elles sont à plat ou en position verticale entre les cailloux. Les sables grossiers et les granules contiennent d'abondants fragments d'os brûlés. Un limon sableux brun rouge foncé colmate partiellement la porosité. L'ensemble du dépôt est induré de façon plus ou moins complète par de la calcite.

9 La position topographique du secteur GPA a favorisé un lessivage partiel du niveau du Gravettien par les eaux de ruissellement. Ce phénomène a laissé sur place de très nombreux petits débris d'os brûlés, piégés entre les éléments grossiers (cailloux calcaires, ossements...). En revanche, compte tenu de la pente et de la rugosité de la surface du dépôt, les écoulements n'ont probablement pas été assez compétents pour entraîner et déplacer la mandibule humaine. La couche archéologique a également pu être affectée par les inondations de la salle; cette hypothèse demande encore à être discutée après avoir comparé les altitudes de la couche archéologique et du niveau d'ennoiement visible au pied de la paroi ornée. La cimentation partielle du sédiment est post-dépositionnelle et correspond à la phase préliminaire de constitution du plancher sus-jacent.

Figure 1 - Carte de localisation des principaux sites gravettiens du Sud-Ouest français ayant livré des vestiges humains.

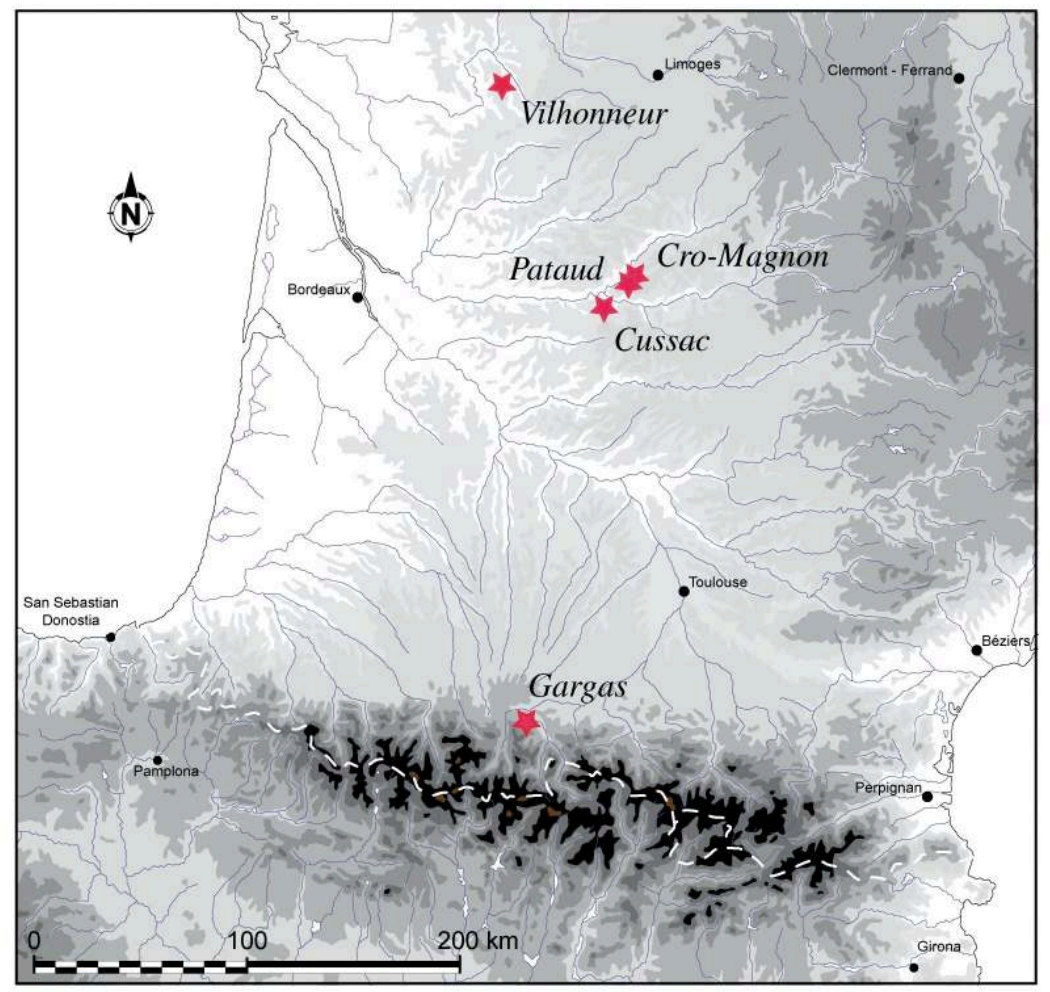




\section{3 - Contexte chrono-stratigraphique de la mandibule}

10 La mandibule a été dégagée au cours du 7e décapage dans la couche 2 (à environ $12 \mathrm{~cm}$ sous le plancher), dans le sous-carré $\mathrm{Wb}^{2}$. Elle se présentait par la face latérale gauche orientée N-S; la partie gauche (corps et branche) reposait à plat sur un bloc décimétrique, auquel elle était soudée par une mince couche de sédiment concrétionné (fig. 5 et 6).

11 L'environnement proche de la mandibule est constitué principalement d'une accumulation de vestiges de faune (esquilles de plusieurs modules et éléments brûlés de petite taille) dont certains portent des traces d'origine anthropique (traitement de boucherie), de quelques éléments de matériel lithique (outils et produits de débitage en silex et quartzite), de galets utilisés et de matières colorantes. À l'exception d'un fragment de clavicule humaine ( $\mathrm{n}^{\circ} 610 \mathrm{~Wb}$ décap 6bis), situé à une trentaine de centimètres de la mandibule, mis au jour dans le décapage précédent, aucun autre vestige humain n'a été découvert sur la surface fouillée ${ }^{3}$, soit environ $2 \mathrm{~m}^{2}$.

Pour l'instant, aucune structuration de l'espace en fonction d'activités précises n'est évidente : pas de structure de combustion, par exemple, mais de nombreux rejets de foyers constitués d'os brûlés, de blocs et de galets calcinés, sont mêlés aux autres vestiges. Cet ensemble stratigraphique semble correspondre à un palimpseste d'occupations domestiques répétées. Par ailleurs, des traces d'activités de préparation sur place de pigments (à base d'oxydes de fer et de manganèse) ont été également enregistrées depuis le premier décapage.

13 Compte tenu de sa position dans un sédiment induré, au sein d'une couche d'habitat scellée anciennement par un plancher stalagmitique et datée par son contexte archéologique $^{4}$, l'attribution de la mandibule au Gravettien semble assurée (fig. 5). L'hypothèse $d u$ creusement postérieur d'une fosse atteignant cette couche pour l'inhumation d'un jeune enfant ne peut être retenue ici' ${ }^{5}$. D'une part, nous n'avons constaté aucune modification dans l'intégrité du plancher stalagmitique recouvrant la zone fouillée (ni trace de creusement, ni reprise de concrétionnement postérieur) et, d'autre part, dans le cas d'une inhumation, il est vraisemblable que d'autres vestiges humains auraient accompagné la mandibule. 
Figure 2 - Plan de la grotte de Gargas avec l'emplacement des panneaux ornés, des fouilles anciennes et récentes (d'après Barrière 1984 ; modifié Foucher, Texier 2004).

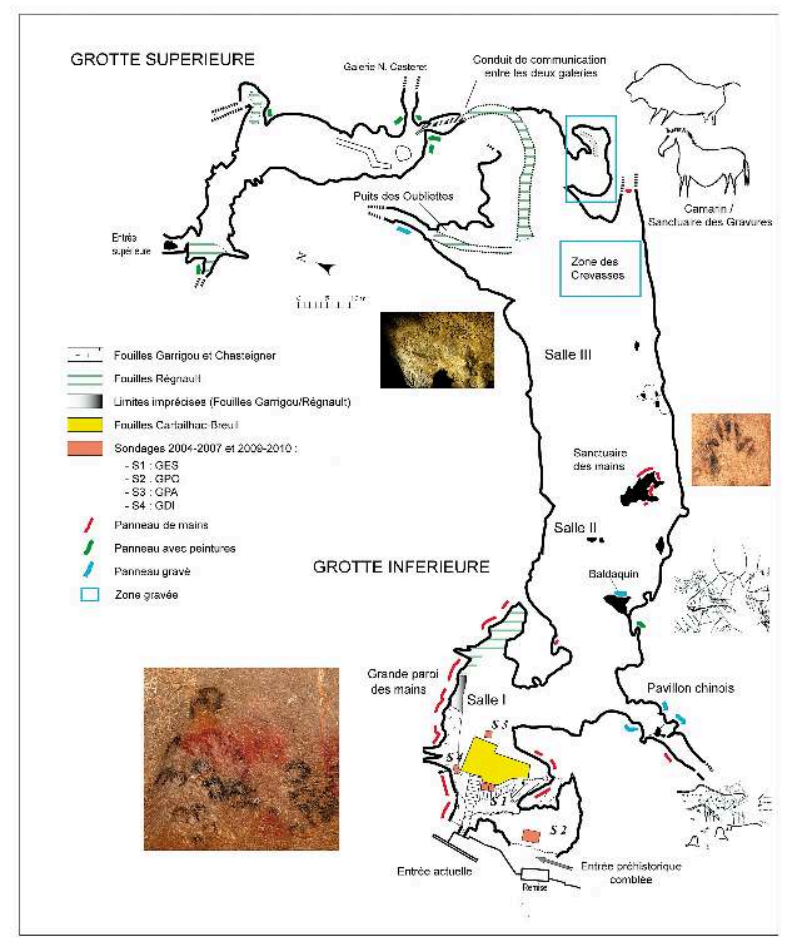

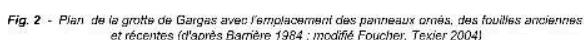

Figure 3 - Localisation du sondage GPA dans la Salle I - Grande paroi des Mains (photo de J.F. Peiré - Drac Midi-Pyrénées).

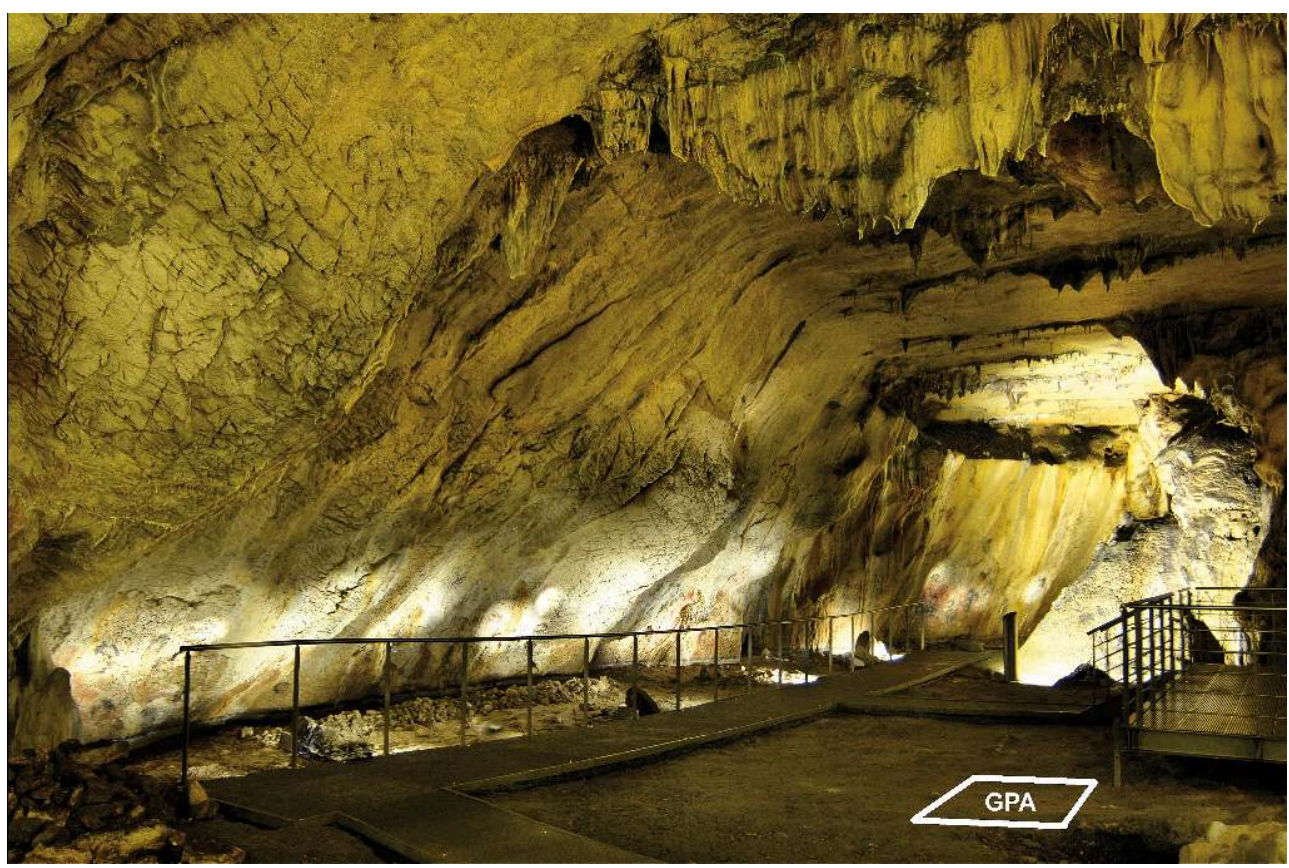




\section{4 - Description préliminaire de la mandibule}

Lors de sa découverte, la mandibule a été laissée in situ pour permettre la réalisation des observations anthropologiques de terrain (photographies, analyses préliminaires et descriptions du contexte immédiat). Puis, son prélèvement, assuré par l'un de nous (D. H.-G.), a été effectué selon le protocole en vigueur pour la recherche d'ADN (conditions stériles maximales pour éviter toute contamination supplémentaire : port de blouse, charlotte, masque, gants, outils désinfectés...). La mandibule a donc été conditionnée dans un environnement stérile et déposée dans une glacière, jusqu'à son transport au laboratoire de restauration Materia Viva (Toulouse, dépôt de Délicieux) où elle a été conservée dans une chambre froide. Marie-France Deguilloux (MC-PACEA, université Bordeaux 1, responsable de la plateforme $\mathrm{ADN}$ ) a réalisé le prélèvement $\mathrm{ADN}$ dans la portion postérieure du corps droit. L'étude paléogénomique est en cours.

Cette mandibule n'a pas encore été séparée du bloc auquel elle est liée par des concrétions. Cette opération débutera dès que l'acquisition et la segmentation des données microtomographiques seront disponibles et les informations restent très préliminaires.

L'examen en l'état montre qu'il s'agit d'une mandibule incomplète de sujet immature (entre deux et cinq ans). Elle comprend la partie gauche du corps sans dent allant de la symphyse à la branche mandibulaire ( ${ }^{\circ} 646$ a) et le corps droit $\left(n^{\circ} 646\right.$ b), préservé sur 2-3 cm. Ce dernier, cassé anciennement à hauteur $\mathrm{de} d \mathrm{~m}_{2} / \mathrm{M}_{1}$ porte la première molaire déciduale inférieure (fig. 7 et 8). Il est séparé de la région symphysaire par une fissure allant du bord alvéolaire au bord inférieur (fig. 8). Le condyle gauche semble intact. Le processus coronoïde gauche, le bord inférieur de l'angle de la branche gauche et de la région symphysaire sont endommagés (fig. 7).

Une fine pellicule de concrétion recouvre la surface de la branche gauche, une partie du corps gauche, la symphyse et la totalité du bord alvéolaire conservé. Là où il est apparent, l'os paraît peu dégradé (fig. 7 et 8).

Sur la face latérale et sous l'angle entre corps et branche gauche apparaît un renflement anormal dont il faudra préciser la nature (lésion pathologique ou altération taphonomique: fig. $7 b$ ). Aucune trace d'ocre n'est visible sur la surface actuellement dégagée. 
Figure 4 - Gargas Secteur GPA. Les écoulements d'eau superficiels sont à l'origine de gours étagés sur le sol de la galerie menant à la partie profonde de la grotte (a). L'eau infiltrée par les fissures du plafond alimente, par ailleurs, une concrétion stalagmitique (b) prolongée par un plancher (c) qui a recouvert le niveau du Gravettien (d). Dans la zone des fouilles anciennes, sous le plancher (c) subsistent encore des lambeaux en place du niveau Gravettien (e).

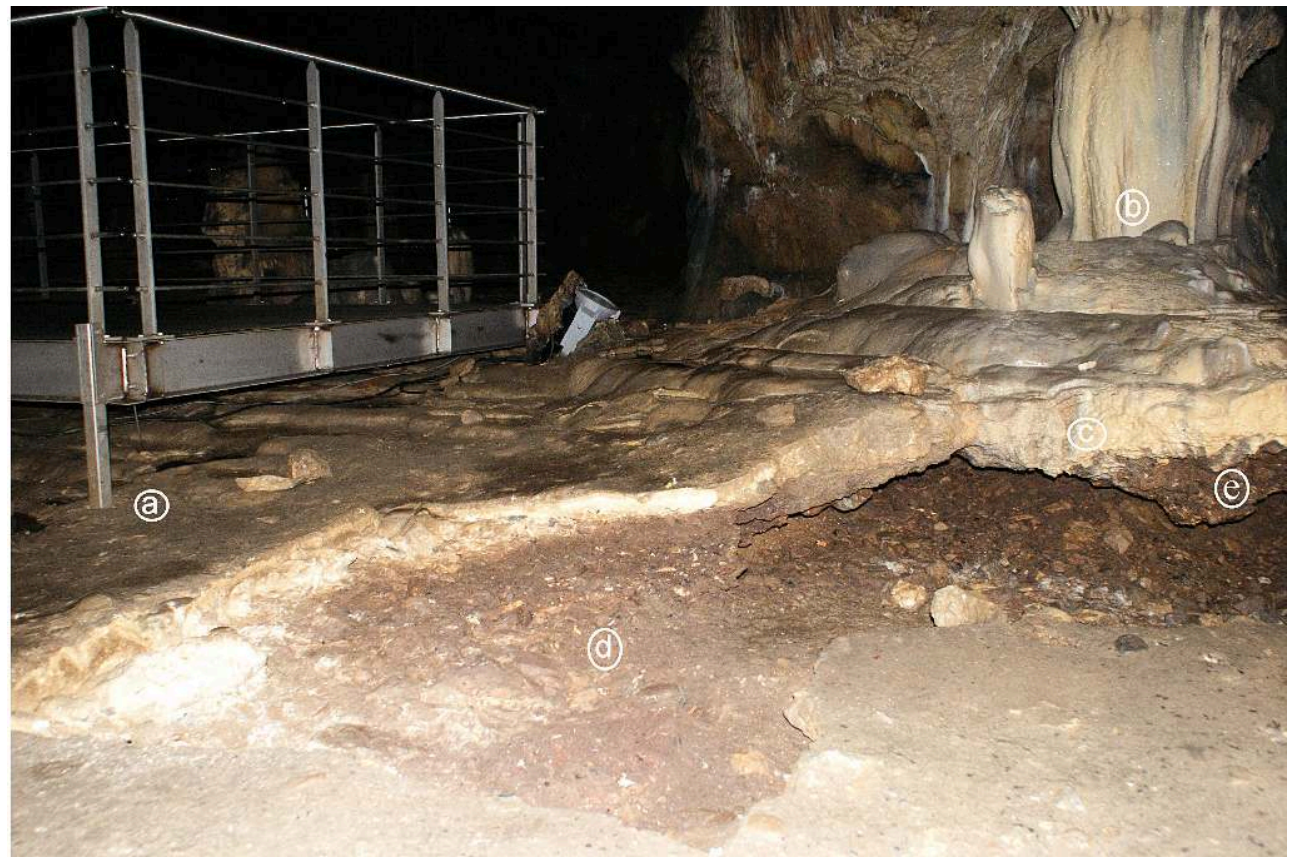

Figure 5 - Gargas sondage GPA. Industrie lithique, parure et galets-broyeurs de l'occupation gravettienne.

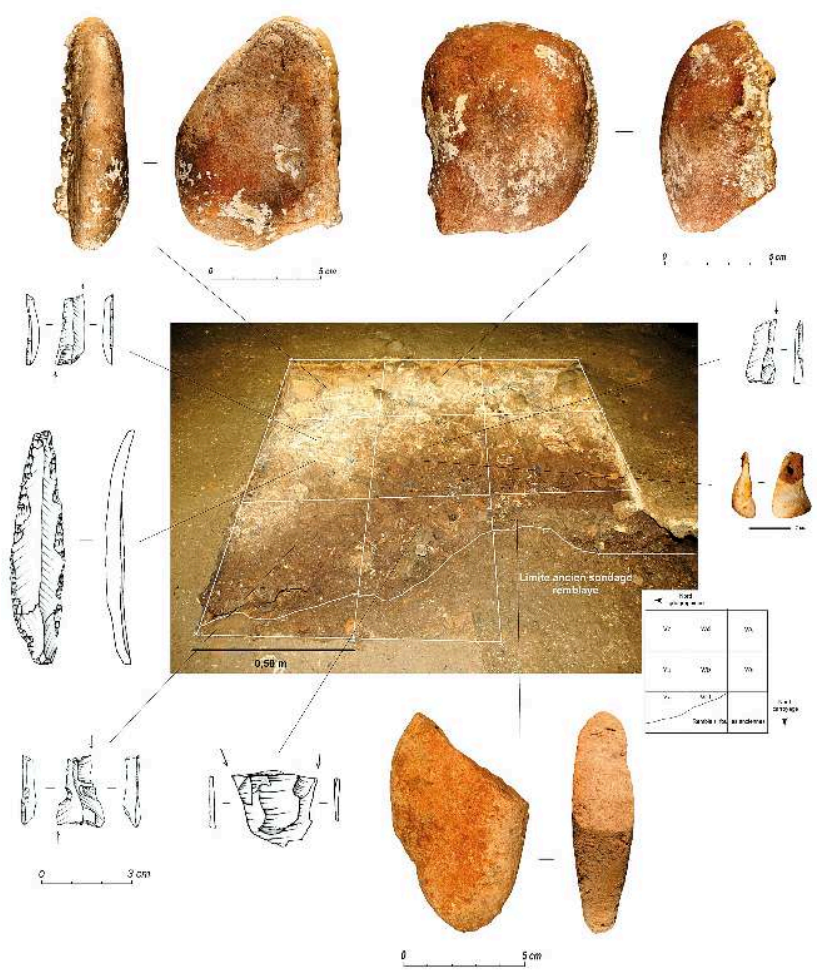


Figure 6 - Gargas Sondage GPA (photos P. Foucher). Carré Wb, niv. 2, décapage 7 et vue en détail de la mandibule $\mathrm{n}^{\circ}(646)$, au moment de sa découverte.

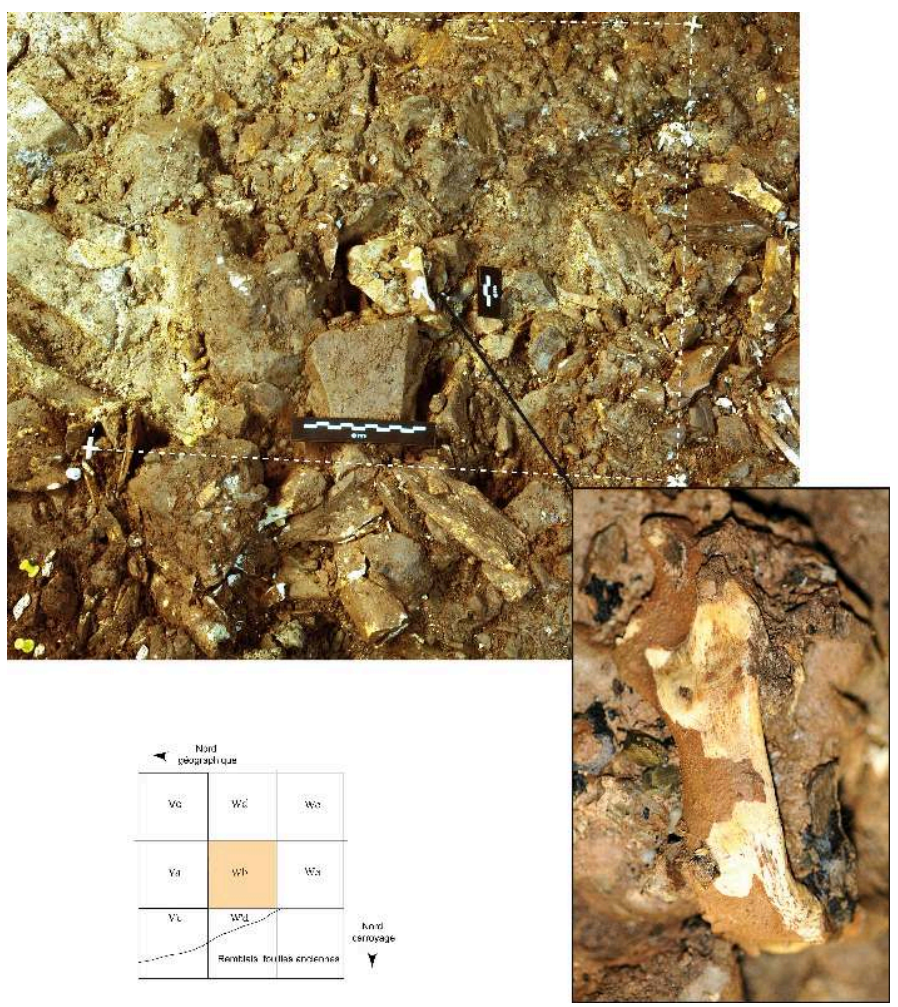

Figure 7 - Gargas Secteur GPA. Carré Wb : niv. 2, décapage 7 (photos D. Henry-Gambier et $P$. Foucher). (a) Vue rapprochée de la mandibule en place ( ${ }^{\circ} 646$ a). $1:$ symphyse. $2:$ condyle. 3 : emplacement d'une éventuelle anomalie sur le corps gauche. (b) Détail de l'anomalie.
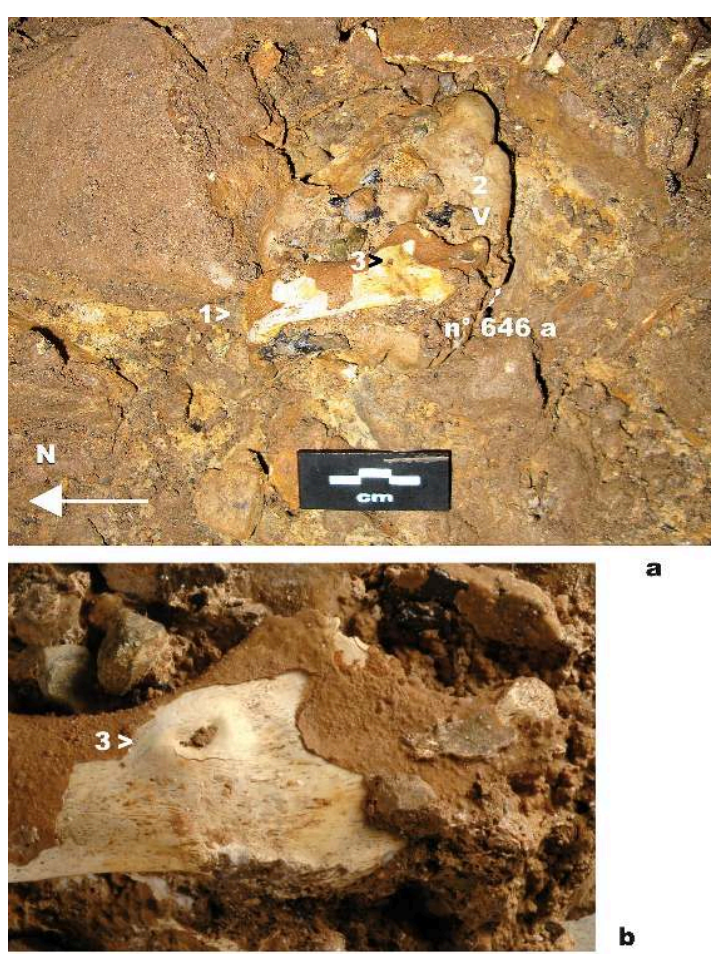
Figure 8 - Gargas Secteur GPA. Carré Wb : niv. 2, décapage 7 (photo D. Henry-Gambier). Vue antérieure de la mandibule. 1 : fissure qui sépare la mandibule en deux fragments. 2 : symphyse.

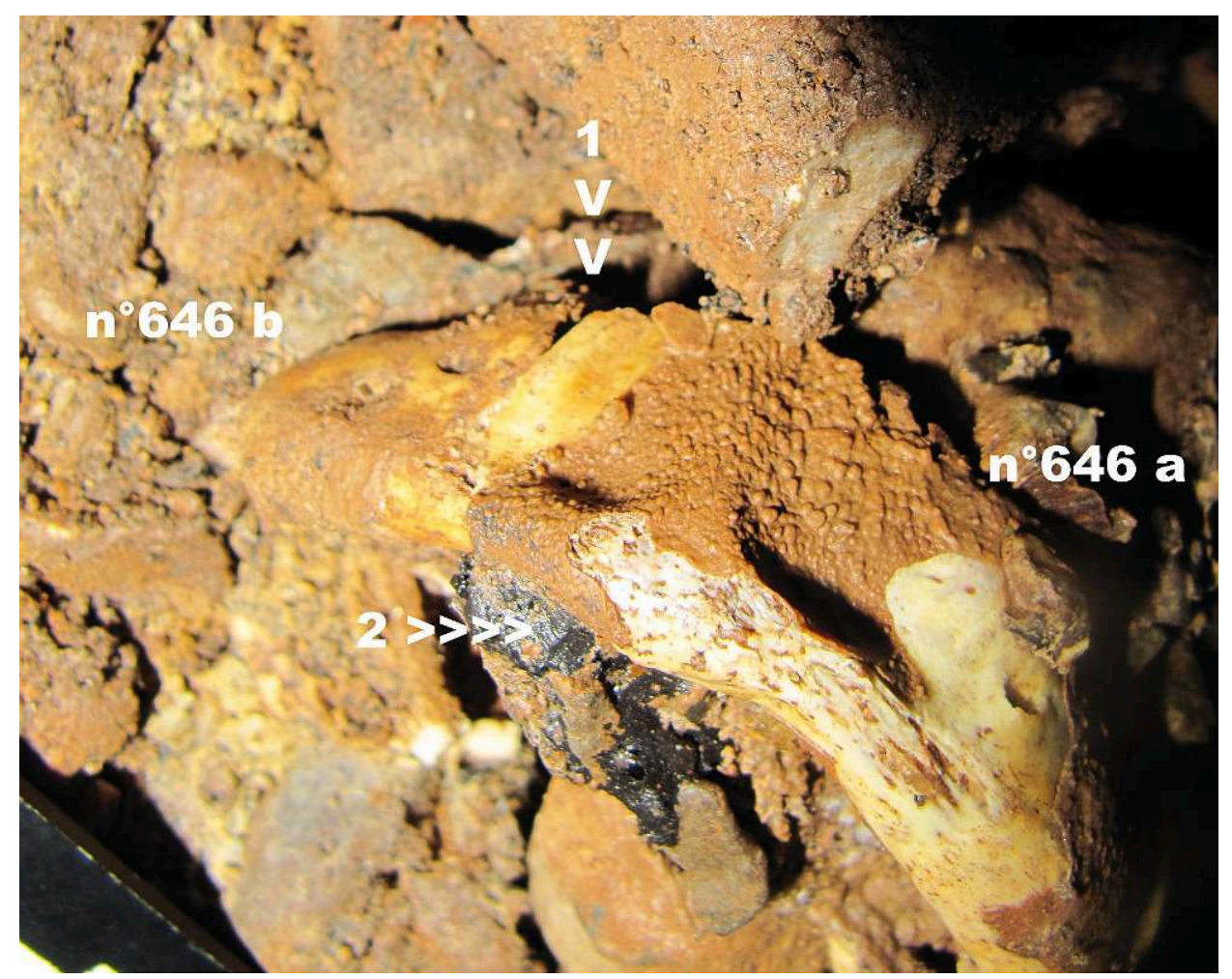

\section{5 - Intérêt scientifique du vestige découvert}

Dans l'état actuel des recherches, il s'agit d'un reste isolé comme cela est fréquent en contexte du Paléolithique supérieur, quel que soit le techno-complexe associé. Toutefois cette découverte est importante à plusieurs titres :

1. À l'exception d'un fragment de fémur signalé par H. Obermaier (1925) et repris par Vallois et Movius (1952), dont la localisation d'origine n'est pas connue, la galerie inférieure de Gargas n'avait jusqu'à présent livré aucun vestige humain.

2. Sa position stratigraphique implique son appartenance au Gravettien à burin de Noailles. Elle contribue ainsi à enrichir le corpus des vestiges humains de cette période, finalement assez pauvre en France et notamment dans les Pyrénées, où seule la grotte d'Isturitz (Pyrénées-Atlantiques) aurait livré anciennement quelques vestiges dans un contexte chronoculturel contemporain (sensu lato). La France ne compte que quatre sites gravettiens ayant livré des vestiges humains significatifs (squelettes relativement bien conservés) (fig. 1): Cro-Magnon, Cussac et Pataud en Dordogne, Vilhonneur en Charente. Ailleurs, les découvertes se limitent à de rares dents isolées ou à de petits fragments osseux peu informatifs (Henry-Gambier et al. 2007; Henry-Gambier 2008; Henry-Gambier et al. s-p a et b). La mandibule de Gargas est suffisamment bien conservée pour apporter de nouvelles données (paléogénétiques et morphométriques) sur les populations de cette culture et en particulier sur les jeunes enfants (Henry-Gambier 2001).

3. Les données actuelles indiquent que cette mandibule n'est pas en position primaire. La poursuite de l'analyse de la surface de cette pièce (recherche des dégradations et des altérations ${ }^{6}$ ), de l'étude géoarchéologique du niveau tel qu'il apparait à travers la fenêtre de lecture que constitue le sondage, ainsi que les autres données archéologiques seront essentielles pour discuter de la signification de ce vestige et des raisons de sa présence dans 
la couche 2. Les investigations en cours devraient permettre de déterminer si ce reste humain provient du démantèlement naturel ou anthropique d'une sépulture, ou s'il ne s'agit que d'un vestige isolé et, dans ce cas, de quel comportement témoigne-t-il ?

20 En effet, il faut rappeler que, pour le Gravettien européen, la très grande majorité des vestiges humains a été découverte en contexte sépulcral et l'inhumation est, en l'état actuel de nos connaissances, la pratique funéraire la mieux documentée (HenryGambier 2008). Sur le territoire français, sans entrer dans le détail des résultats des études en cours, il apparaît que le contexte et les caractéristiques des dépôts de corps à Cro-Magnon, Cussac, Pataud et Vilhonneur se distinguent de ceux décrits pour le reste de l'Europe (Aujoulat et al. 2002 ; Henry-Gambier et al. 2007 ; Henry-Gambier et al. s-p a et b). En d'autres termes, sur le territoire français, l'inhumation ne semble pas pour l'instant constituer la pratique retenue par les gravettiens. En outre, trois des sites (Cussac, Vilhonneur et Pataud) «associent » vestiges humains et décors pariétaux (Henry-Gambier et al. s-p a et b).

21 Dans la grotte de Vilhonneur, les thématiques pariétales sont peux nombreuses et situées dans la même salle que le squelette humain (Henry-Gambier et al. 2007). Des investigations plus poussées sont cependant nécessaires pour préciser l'éventuelle relation entre le squelette et les peintures.

À Cussac, les trois locus où ont été déposés les corps d'au moins six individus sont localisés dans la galerie aval où se trouvent la majorité des gravures. Mais aucun des locus n'est en relation directe avec un panneau gravé (Aujoulat et al. 2002 ; HenryGambier et al. s-p a). Cependant, comme à Vilhonneur, aucune fouille archéologique des dépôts funéraires n'ayant débutée, il est pour l'instant impossible de statuer sur les liens entre le dépôt des os humains et les figurations pariétales.

23 Sur le site de Gargas, l'analyse du réseau orné permet de considérer deux ensembles pariétaux distincts, du point de vue chronologique et thématique : la Galerie supérieure et la Galerie inférieure. Le dispositif de cette dernière présente une forte structuration topographique, en fonction des motifs représentés (empreintes de mains négatives, bestiaire gravé, tracés digitaux), et son contexte archéologique permet de l'attribuer au Gravettien (Clottes et al. 1992 ; Foucher et al. 2007 ; 2008a). Il faut souligner que la Salle I, proche de l'entrée préhistorique, rassemble les deux tiers des empreintes des mains de Gargas et a été le lieu d'un vaste habitat, fréquenté entre 28000 et 25000 BP (Foucher et al. 2011). Les recherches récentes sur ces occupations indiquent une grande diversité d'activités, pour la plupart en relation avec les exigences de la vie quotidienne: fabrication d'outils, de parures et d'armatures de chasse, traitements des peaux, activités de boucherie et réutilisation des carcasses à des fins non alimentaires (Foucher, San Juan-Foucher dir. 2011 ; Foucher et al. 2008b, 2012). Nous trouvons donc associés, dans un même lieu (la Salle I), un espace "symbolique " et un espace «domestique ». C'est dans ce contexte que se positionne la mandibule d'enfant. En attendant les résultats des études complémentaires (paléogénomie, morphométrie classique et taphonomie), force est de constater que cette nouvelle découverte apporte une composante supplémentaire aux perspectives d'étude et d'interprétation du site de Gargas.

24 Il est d'ailleurs important de noter que, même si à Cussac, Pataud ou Vilhonneur, les vestiges humains sont plus nombreux, la mandibule de Gargas constitue un nouvel exemple de vestige humain trouvé dans une grotte ornée. 
Les récentes découvertes françaises permettent ainsi d'ouvrir un nouveau chapitre sur les relations entre pratiques mortuaires et art pariétal, même s'il faut préciser que chacun de ces sites présente des contextes pariétaux et des structurations spatiales très divers, qu'il faudra prendre en compte au moment des interprétations. Ces découvertes contribueront sans doute à préciser nos connaissances sur les comportements autour de la mort dans la culture gravettienne, notamment dans le sud-ouest de l'Europe.

Les recherches en cours dans la grotte de Gargas se font dans le cadre d'une opération programmée pluriannuelle, financée par le Ministère de la Culture et de la Communication, DRAC-Midi-Pyrénées, avec le soutien du Conseil général des Hautes-Pyrénées. L'association Archéologies est également partenaire de cette opération. Nous remercions particulièrement la municipalité d'Aventignan, propriétaire de la grotte, pour son soutien logistique et les facilités accordées à notre projet.

\section{BIBLIOGRAPHIE}

AUJOULAT N., GENESTE J.-M., ARCHAMBEAU Ch., BARRAUD D., DELLUC M., DUDAY H., HENRYGAMBIER D. 2002 - La grotte ornée de Cussac - Le Buisson-de-Cadouin (Dordogne) : premières observations. Bulletin de la Société Préhistorique Française, 99 (1), p. 129-137.

BOULESTIN B. 1999 - Approche taphonomique des restes humains. Le cas des Mésolithiques de la grotte des Perrats et le problème du cannibalisme en préhistoire récente européenne. Oxford: Archaeopress (BAR International Series, 776), $276 \mathrm{p}$.

BREUIL H., CHEYNIER A. 1958 - Les fouilles de Breuil et Cartailhac dans la grotte de Gargas en 1911 et 1913. Bulletin de la Société méridionale de spéléologie et de préhistoire, V, 1954-55, p. 341-382 (extrait du Bulletin de la Société d'histoire naturelle de Toulouse, 1958, 93).

CLOTTES J., VALLADAS, H. CACHIER, H., ARNOLD, M. 1992 - Des dates pour Niaux et Gargas. Bulletin de la Société Préhistorique Française, 89 (9), p. 270-274.

FOUCHER P. 2004 - Les industries lithiques du complexe Gravettien-Solutréen dans les Pyrénées. Technotypologie et circulation des matières siliceuses de part et d'autre de l'axe Pyrénées-Cantabres. Thèse de l'Université de Toulouse 2-le-Mirail, 3 vol. , 647 p.

FOUCHER P. 2006 - Gargas et l'Atlantique : les relations transpyrénéennes au cours du Gravettien. In : Homenaje al Prof. Jesús Altuna. Munibe, 57, 2005-2006, tome II : Arqueología, p. 131-147.

FOUCHER P. à paraître - Synthèse chrono-culturelle sur le Gravettien des Pyrénées : constat et réflexions sur la stabilité régionale des traditions techniques. In : El Gravetiense cantábrico, octobre 2011, Museo de Altamira.

FOUCHER P., SAN JUAN-FOUCHER C. (dir.), avec la collaboration de ANGÁS PAJAS J., FERRIER C., M.-F. DEGUILLOUX, DRIEUX-DAGUERRE M., D. HENRY-GAMBIER, IRIARTE CHIAPUSO M.-J., MIRANDA OLIVÁN J., MOURRE V., PEYROUX M., POPLIN F., OBERLIN Ch., QUEFFELEC A., SÉRONIEVIVIEN M.-R., SERVELLE Ch., VERCOUTÈRE C. 2011. - La grotte de Gargas (Aventignan, HautesPyrénées) : rapport de synthèse de fouille programmée (triennale 2009-2011). Toulouse : Service Régional de l'Archéologie de Midi-Pyrénées, 185 p. 
FOUCHER P., SAN JUAN-FOUCHER C., OBERLIN C. 2011 - Les niveaux d'occupation gravettiens de Gargas (Hautes-Pyrénées) : nouvelles données chronostratigraphiques. In : N. Goutas, L. Klaric, D. Pesesse, P. Guillermin (dir.), À la recherche des identités gravettiennes : actualités, questionnements et perspectives. Actes de la table ronde sur le Gravettien en France et dans les pays limitrophes, Aixen-Provence, 6-8 octobre 2008, Éd. Société préhistorique française, p. 373-385 (Mémoire LII).

FOUCHER P., SAN JUAN-FOUCHER C., RUMEAU Y. - 2007 - La grotte de Gargas. Un siècle de recherches. Communauté de communes du canton de Saint-Laurent-de-Neste, $126 \mathrm{p}$.

FOUCHER P., SAN JUAN-FOUCHER C., RUMEAU Y. 2008a - La grotte de Gargas : un sanctuaire préhistorique au cœur des Pyrénées. Archéologia, 459 (oct.), p. 44-51.

FOUCHER P., SAN JUAN C., SACCHI D., ARRIZABALAGA A. 2008b - Le Gravettien des Pyrénées. In : Le Gravettien : entités régionales d'une paléoculture européenne. Actes du colloque des Eyzies, juillet, 2004, Paleo, 20, p. 331-356.

FOUCHER P., SAN JUAN-FOUCHER C., VERCOUTÈRE C., FERRIER C. 2012 - La grotte de Gargas (Hautes-Pyrénées, France) : l'apport du contexte archéologique à l'interprétation de l'art pariétal. In : J. Clottes (dir), L'art pléistocène dans le monde / Pleistocene art of the world / Arte pleistoceno en el mundo. Actes du Congrès IFRAO, Tarascon-sur-Ariège, 6-11 septembre 2010. $\mathrm{N}^{\circ}$ spécial de Préhistoire, Art et Sociétés, Bulletin de la Société Préhistorique Ariège-Pyrénées, LXV-LXVI, 2010-2011, livre : p. 52-53 et CD : p. 209-225.

FOUCHER P., SAN JUAN-FOUCHER C., FERRIER C., COUCHOUD I., VERCOUTÈRE C. 2008c - La grotte de Gargas (Aventignan, Hautes-Pyrénées) : nouvelles perspectives de recherche et premiers résultats sur les occupations gravettiennes. In : J. Jaubert, J.-G. Bordes, I. Ortega (dir.), Les sociétés paléolithiques d'un grand Sud-Ouest - Nouveaux gisements, nouvelles méthodes, nouveaux résultats. Actes des journées de la Société préhistorique française et de l'Université de Bordeaux I, nov. 2006, p. 301-324 (Mémoire de la Société préhistorique française, 47).

FOUCHER P., VALLADAS H., TISNERAT N., DUDAY H., GACHINA J. 1995 - Le squelette réputé aurignacien de la grotte du Bouil Bleu à la Roche Courbon (Saint-Porchaire, Charente-Maritime) : révision de l'âge-datation directe par la méthode du carbone 14 (S.M.A.). Bulletin de la Société préhistorique française, 92 (4), p. 443-444.

HENRY-GAMBIER D. 2001 - Les enfants de Grimaldi (Grotte des Enfants site des Baoussé - Roussé, Italie). Anthropologie et Palethnologie funéraire. CTHS/RMN. 250 p.

HENRY-GAMBIER D. 2008 - Pratiques funéraires et comportements des populations gravettiennes en Europe : bilan des données et interprétations. Paléo, 20, p. 399-438.

HENRY-GAMBIER D., BEAUVAL C., AIRVAUX J., AUJOULAT N., BARATIN J.F., BUISSON-CATIL J. 2007 - New hominid remains associated with gravettian parietal art (Les Garennes, Vilhonneur, France). Journal of Human Evolution, 53 (6), p. 747-750.

HENRY-GAMBIER D., COURTAUD P., DUDAY H., DUTAILLY B., VILLOTTE S., DEGUILLOUX MF., PÉMONGE MH., AUJOULAT N., DELLUC M., FOURMENT N., JAUBERT J. sous-presse (a) - Grotte de Cussac (Le Buisson-de-Cadouin) : un exemple de comportement original pour le Gravettien. Acte du Congrès de la SPF - Les-Eyzies- de Tayac (Dordogne) juin 2010.

HENRY-GAMBIER D., VILLOTTE S., BEAUVAL C., BRUZEK J., GRIMAUD-HERVÉ D. sous-presse (b) Les vestiges humains : un assemblage original. In : Le Gravettien final de l'abri Pataud (Dordogne, France) Fouilles et études 2005 - 2009 R. Nespoulet, L. Chiotti, D. Henry - Gambier (Dir) BAR International Series XXXX, 2012 p. 135-176. 
OBERMAIER H. 1925 - El hombre fósil. Madrid : Museo de Ciencias naturales, 2e Ed. Comisión de Investigaciones paleontológicas y prehistóricas, 457 p. (Memoria 9).

SAN JUAN-FOUCHER C. 2011 - Industrie osseuse décorée et parures gravettiennes de Gargas (Hautes-Pyrénées, France) : marqueurs culturels, sociaux et territoriaux. In : N. Goutas, L. Klaric, D. Pesesse, P. Guillermin (dir.), À la recherche des identités gravettiennes : actualités, questionnements et perspectives. Actes de la table ronde sur le Gravettien en France et dans les pays limitrophes, Aix-en-Provence, 6-8 octobre 2008, Éd. Société préhistorique française, p. 225-241 (Mémoire LII).

SAN JUAN-FOUCHER C. à paraître - Industria ósea decorada y arte mueble del gravetiense pirenaico : perspectivas territoriales actualizadas. In : El Gravetiense cantábrico, octobre 2011, Museo de Altamira.

VALLOIS H.V., MOVIUS H. L. 1952 - Catalogue des hommes fossiles. In : XIXe congrès géologique international, Alger, fasc. V, $378 \mathrm{p}$.

\section{NOTES}

1. Pour un historique complet des recherches sur la grotte, se reporter à Foucher 2004 et Foucher et al. 2007.

2. La mandibule a été découverte dans l'après-midi du vendredi 16 septembre 2011 et porte le $\mathrm{n}^{\circ}$ d'inventaire : GPA-11-Wb-646.

3. Le matériel osseux issu des décapages 6 bis et 7 a été entièrement révisé conjointement par D. H.-G. et C. V.

4. Le matériel lithique a livré des burins de Noailles dans tous les décapages successifs (1 à 7 ).

5. Cas de figure bien reconnu à la grotte du Bouil Bleu de la Roche Courbon (Saint-Porchaire, Charente maritime) : cf. Foucher et al. 1995.

6. Dégradations : ensemble des processus essentiellement détériorants, voire destructeurs, qui tendent à masquer ou supprimer des informations. Altérations : modifications qui transforment l'os par rapport à son état normal et sont génératrices d'informations. Elles sont généralement circonscrites et n'interviennent pas sur les propriétés des os (Boulestin 1999 - p. 45).

\section{RÉSUMÉS}

La Galerie inférieure de la grotte de Gargas (Hautes-Pyrénées) fait l'objet de nouvelles recherches archéologiques depuis 2004. Les principaux résultats obtenus concernent les occupations gravettiennes de cette galerie et ont permis une réactualisation des données anciennes des fouilles d'É. Cartailhac et de H. Breuil (1911-1913), en particulier celles relatives aux caractéristiques fonctionnelles, spatiales et chronologiques de la fréquentation du site par les auteurs des manifestations pariétales. Au cours de la campagne 2011, une mandibule de jeune enfant a été découverte dans la partie supérieure du niveau 2 de la Salle I, lieu où se trouvent les deux tiers des empreintes de mains négatives connues dans ce site. L'objet de cet article est de rendre compte de cette découverte, par une description préliminaire de la mandibule et de sa position stratigraphique. L'intérêt de ce vestige humain est évalué par rapport au contexte des découvertes récentes faites à Cussac, à Vilhonneur et aux données réactualisées pour le sud-ouest 
de l'Europe. L'éventuelle existence d'un contexte funéraire à Gargas pourrait faire évoluer les perspectives d'étude et d'interprétation de ce site de référence du Gravettien européen.

Since 2004, new archaeological researches have been carried out within the lower gallery of the Gargas cave (Hautes-Pyrénées). The main results involved the Gravettian settlements of this gallery and allowed to update the former data of the excavations managed by É. Cartailhac and H. Breuil (1911-1913) : in particular, data about functional, spatial and chronological characteristics of the use of site by people who made paintings. During the 2011 excavation, a mandible of a young child was discovered in the upper part of the Gravettian level of the room I, where are also located the two thirds of the painted hands. The topic of this paper is to underline this discovery with a preliminary description of this mandible and of its stratigraphical location. The interest of this human remain is evaluated in regard to the context of the recent discoveries in Cussac and Vilhonneur and to the updated data for the southwestern Europe. The possible existence of a funerary context in Gargas cave could make the further studies and interpretations evolved for this key site of the Gravettian in Europe.

\section{INDEX}

Mots-clés : Gravettien, Homo sapiens sapiens, Pyrénées

Keywords : Gravettian, Homo sapiens sapiens, Pyrenees

\section{AUTEURS}

\section{PASCAL FOUCHER}

Service Régional de l'Archéologie - DRAC Midi-Pyrénées, rue de la Dalbade, 31000 Toulouse et UMR 5608 TRACES - Université de Toulouse-le-Mirail, 5 allées Antonio Machado, 31000 Toulouse, France - cristina.sanjuan@culture.gouv.fr ;pascal.foucher@culture.gouv.fr

\section{CRISTINA SAN JUAN-FOUCHER}

Service Régional de l'Archéologie - DRAC Midi-Pyrénées, rue de la Dalbade, 31000 Toulouse et UMR 5608 TRACES - Université de Toulouse-le-Mirail, 5 allées Antonio Machado, 31000 Toulouse, France - cristina.sanjuan@culture.gouv.fr ;pascal.foucher@culture.gouv.fr

\section{DOMINIQUE HENRY-GAMBIER}

CNRS, PACEA/A3P, UMR 5199, avenue des Facultés, 33405 Talence cedex, France d.gambier@pacea.u-bordeaux1.fr

\section{CAROLE VERCOUTÈRE}

UMR 7194, Département de Préhistoire, Muséum national d'Histoire naturelle,Paris, France cvercout@mnhn.fr

\section{CATHERINE FERRIER}

Université de Bordeaux, PACEA/PPP, UMR 5199, avenue des Facultés, 33405 Talence cedex, France - c.ferrier@ipgq.u-bordeaux1.fr 